\title{
On Simulation Modeling of Information Dissemination Systems in Mobile Environments
}

\author{
Wang-Chien Lee, Johnson Lee, and Karen Huff \\ GTE Laboratories Incorporated, 40 Sylvan Road, Waltham, MA 02451, USA \\ $\{$ wlee, jlee, khuff\}@gte.com
}

\begin{abstract}
In this paper, we propose dynamic discrete-event based models for information dissemination systems in both single cell and multiple cell mobile environments. We demonstrate that this novel approach can flexibly model channel borrowing and hand-off scenarios. Finally, we implement two simulation systems based on the single cell model. Our simulations show that broadcast channels can effectively alleviate traffic overload in a cell.
\end{abstract}

\section{Introduction}

In the forthcoming era of ubiquitous information services, wireless bandwidth is one of the most valuable resources. How to utilize the limited wireless bandwidth to provide a wide range of services for mobile users is a critical research issue.

Cell splitting and information broadcasting are two of the techniques for alleviating demands for wireless bandwidth. Cell splitting is to divide a large cell into a number of smaller cells. By reducing the size of the cells, more cells per area will be available. Thus, the number of channels and traffic capability in the area is increased. On the other hand, information broadcasting is very useful for disseminating data of common interest, e.g., stock ticks and breaking news, to a large population of mobile users. By dedicating some channels for broadcasting popular information, overloaded traffic on traditional point-to-point, ondemand channels may be alleviated. Moreover, broadcast channels are a natural approach for realization of push-oriented information dissemination which balances pull-based data access on point-to-point channels. As a result, research and new developments in mobile information systems have involved both broadcast channels and point-to-point channels as communication media for wireless information dissemination systems [V94 AAFZ95 LHL99 SRB97]. In this paper, we consider performance modeling of information dissemination systems in single and multiple cell mobile environments.

An interesting and important aspect of mobile computing in multiple cell environments is roaming. In order to facilitate wireless roaming, base stations have to negotiate with each other to ensure continuous service to a user moving across cells. Whenever a mobile user moves into a new cell, his mobile computer registers with the base station in charge of that cell. This registration process usually involves communication between the old base station and the new base station to hand-off information associated with the mobile user and computer.

H.V. Leong et al. (Eds.), MDA'99, LNCS 1748, pp. 45 571999.

(C) Springer-Verlag Berlin Heidelberg 1999 
During off-peak hours, base stations usually have light competition for channels and thus can ensure a smooth hand-off for the roaming users. During peak hours, however, base stations may have too many mobile users waiting for channels to be available. In this situation, the base station may adopt a policy to assign higher priorities to the already connected, roaming users with a hope that channels can be allocated for the roaming users before they enter the cell. Research on supporting communication hand-off has been presented in [BB95 TJ91.

In addition to the issue with hand-off, channel borrowing is another interesting aspect of mobile computing which appears in multiple cell environments. Basically, wireless channels are allocated to the cells based on geographic distribution of the traffic load. If some cells become more overloaded than others, it may be possible to reallocate channels by transferring frequencies from the lightly loaded cells to the heavily loaded cells. This is an interim solution, because the borrowed frequencies (and channels) have to be returned to the original cells when the traffic in the original cells grows.

Performance analysis of the mobile computing systems is a critical task for planning and operation of the systems. Queueing theory based models for performance analysis of the mobile systems have appeared in the literature [LHL99 IB94, mostly in the context of single cell mobile computing environments. These queueing models, assuming Poison and Exponential distributions for inputs and service time, are not faithful to the dynamic real world situations. Moreover, these models do not provide global analysis of systems involving crosscell activities. With increasing offerings of ubiquitous services and demands for wireless bandwidth, performance analysis and global optimization of systems and services in multiple cell mobile environments is critical for success in today's competitive market. In this paper, we propose a simulation model, based on dynamic discrete-event graphs, for a wireless information dissemination system in both the single cell and multiple cell mobile environments.

The rest of the paper is organized as follows. In Section 2, we propose a simulation-based performance analysis model for the information dissemination system in the single cell environment. In Section 3, we extend the model to cover multiple cell mobile computing scenarios, i.e., channel borrowing and hand-off. In Section 4, we describe our implementations and discuss simulation results. Finally, Section 5 concludes the paper.

\section{Dynamic Discrete-Event Modeling}

Queueing theory based models have been used for performance analysis of the mobile systems. In LHL99, for example, queueing models and analytical methods are used for performance evaluation of the point-to-point communication channels and the broadcast channels, respectively. Overall system performance is then obtained by combining weighted results from both channels. One major defect of this approach is the lack of one coherent analytical model for the whole system. To remedy the above problem, we develop in this paper a new simulation 
model for wireless information dissemination based on dynamic discrete-event modeling technique HP89.

Dynamic discrete-event modeling is a simulation modeling technique broadly used in experiment-intensive fields such as Nuclear Physics. Compared to queueing theory based models and simulations, the dynamic discrete-event based model and simulations provides us the following important advantages:

- The logical correctness of the model is easy to verify;

- The simulations are easy to implement;

- The simulations are more realistic since some constraints in the queueing model are relaxed:

- Queueing models assume an infinite duration for the simulation;

- Queueing models assume either Poison or Exponential distributions for parameters in the model;

- Queueing models only handle steady states.

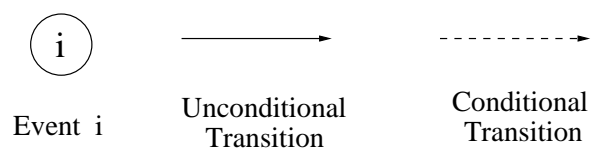

(a)

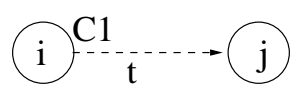

(b)

Fig. 1. Event graph: (a) basic notations; (b) conditional event transition.

Basically, a discrete-event simulation model can be viewed as a model of the interaction of discrete events occurring in the system and the system's state variables. These interactions can be represented as an event graph where the nodes represent the events and the arrows represents transitions between two events. In the graph, a transition between two events can be unconditional or conditional. The time delay and condition for a transition are also denoted in the graph. Figure 1 (a) illustrates the basic notations of the graph and Figure 1(b) implies that providing the necessary condition $\mathrm{C} 1$, event $i$ will lead to event $j$ with a delay of $t$.

In Figure 2 we present an event graph for the wireless information dissemination system in single cell environment. Descriptions of events, conditions and elapsed time in the graph are also given in the following.

\section{EVENTS}

E1: a user attempt to connect to the base station.

E2: blocked user re-send connection request to the base station.

E3: a point-to-point channel allocated and service session begins.

E4: session ends.

E5: user tunes into the broadcast channels. 


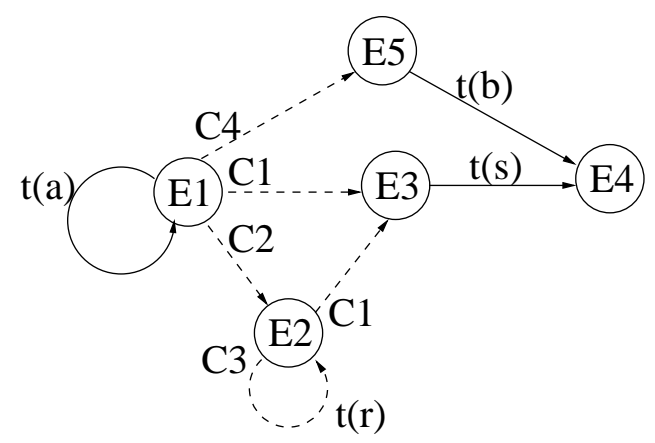

Fig. 2. Single Cell

\section{CONDITIONS}

$\mathrm{C} 1: m<c$.

$\mathrm{C} 2: m=c$.

C3: restless user keeps re-sending connection requests.

C4: user has valid access information for the broadcast channels.

\section{DELAYS}

$\mathrm{t}(\mathrm{a})=$ time until the next user arrives.

$\mathrm{t}(\mathrm{s})=$ service time for a user on a point-to-point channel.

$\mathrm{t}(\mathrm{r})=$ elapsed time for a re-send of connection request.

$\mathrm{t}(\mathrm{b})=$ access time for a user on broadcast channels.

As depicted by the simulation model, a mobile user first tries to connect to the base station and then requests data when he/she enters a cell. If the mobile user has valid access information for the broadcast channels, the mobile user may obtain the data of interests through the broadcast channels. Otherwise, he/she will need to obtain the data through a point-to-point channel. If the traffic is light, the mobile user can easily connect to the base station. However, if the system is overloaded, i.e., all of the available channels are in use, the mobile user will have to re-send the connection requests until one of the other mobile users relinquishes a channel.

One major advantage of this simulation model is that it accurately captures activities of the wireless information dissemination systems. By implementing the model with tools or programs, we can simulate those activities and obtain important information regarding to system characteristics and performance.

\section{Multiple Cell Mobile Environments}

In this section, we extend the event graph of a single cell wireless information dissemination system to cover the scenarios of channel borrowing and hand-off in multiple cell mobile environments. 


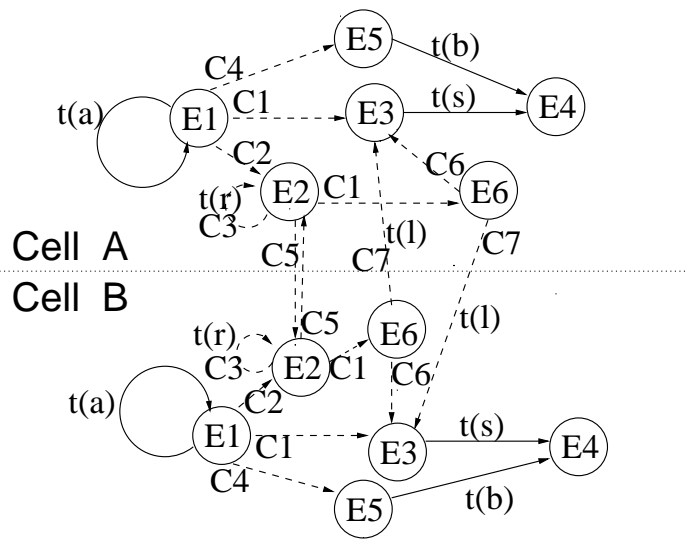

Fig. 3. Channel Borrowing

\subsection{Channel Borrowing}

If some cells become overloaded, it may be possible to re-allocate channels from the lightly loaded cells to the heavily loaded cells. Without losing generality, we present in Figure 3 an event graph for wireless information systems in a two cell mobile environment. The event graph for multiple cell mobile systems can be similarly derived.

\section{EVENTS}

E1: a user attempt to connect to the base station.

E2: blocked user re-sends connection request to the base station.

E3: service session begins.

E4: session ends.

E5: user tunes into the broadcast channels.

E6: point-to-point channel allocated.

\section{CONDITIONS}

$\mathrm{C} 1: m<c$.

$\mathrm{C} 2: m=c$.

C3: restless user keeps re-sending connection requests.

C4: user has valid access information for broadcast channels.

C5: $\left(m=c\right.$ and $\left.m^{\prime}<c^{\prime}\right) \sqrt[1]{1}$ or $\left(m<c\right.$ and $\left.m^{\prime}=c^{\prime}\right)$.

C6: user from the cell.

C7: user from other cell.

\section{DELAYS}

$\mathrm{t}(\mathrm{a})=$ time until the next user arrives.

$\mathrm{t}(\mathrm{s})=$ service time for a user on the point-to-point channel.

$\mathrm{t}(\mathrm{r})=$ elapsed time for a re-send of connection request.

$\mathrm{t}(\mathrm{b})=$ access time for a user on the broadcast channels.

$\mathrm{t}(\mathrm{l})=$ setup time for channel borrowing.

\footnotetext{
${ }^{1}$ Assuming $m^{\prime}$ and $c^{\prime}$ are the number of mobile users and channels in the neighbor cell, respectively.
} 
The basic activities in this multiple cell simulation model are the same as those in the one cell model. However, when all of the available channels in a cell are in use, a mobile user is allowed to obtain a point-to-point channel from the neighbor cell. The steps for channel borrowing are done through negotiation between the two base stations. Thus, the mobile user doesn't know the actions behind the scene. To capture this scenario in the extended multiple cell simulation model, transition arrows between E2 nodes of the neighbor cells are added to the event graph with a condition, C5, which states that all channels in the current cell are in use and that there are free channels in the neighbor cell. Moreover, an event E6 are separated from E3 of the single cell model to denote the situation that a channel has been allocated. The mobile user receives service from the base station of its residential cell. We use $t(1)$ to denote the overhead for channel borrowing.

\subsection{Hand-Off}

Roaming is one of the most important features for mobile systems. Although the mobile users can travel from cell to cell, it is required that a service in progress not be interrupted. Therefore, change of channels and transfer of services due to change of cells, i.e., hand-off, must be conducted transparently. In the following, we again assume a two cell information dissemination system and provide an event graph for the system (see Figure 4). In this model, we assume that both cells are capable of providing the same services.

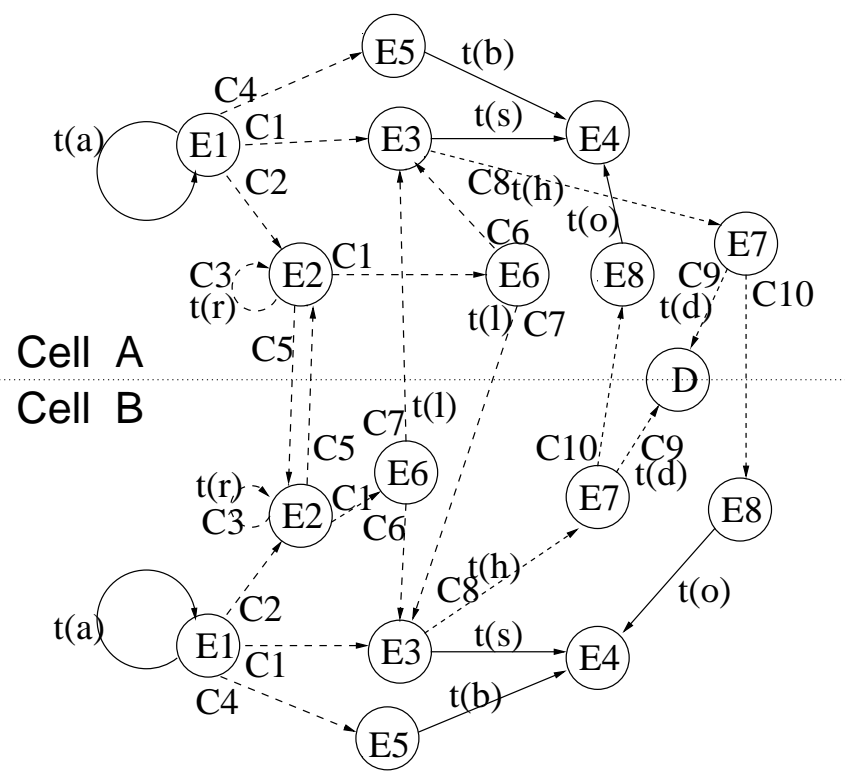

Fig. 4. Hand-off 


\section{EVENTS}

E1: a user attempt to connect to the base station.

E2: blocked user re-sends a connection request to the base station.

E3: service session begins.

E4: session ends.

E5: user tunes into the broadcast channels.

E6: point-to-point channel allocated.

E7: hand-off begins.

E8: hand-off completes.

D: connection drops.

\section{CONDITIONS}

C1: $m<c$.

$\mathrm{C} 2: m=c$.

C3: restless user keeps re-sending connection requests.

C4: user has valid access information for broadcast channels.

C5: $\left(m=c\right.$ and $\left.m^{\prime}<c^{\prime}\right)$ or $\left(m<c\right.$ and $\left.m^{\prime}=c^{\prime}\right)$.

C6: user from the cell.

C7: user from other cell.

C8: roaming users.

C9: hand-off not successful.

C10: hand-off successful.

\section{DELAYS}

$\mathrm{t}(\mathrm{a})=$ time until the next user arrives.

$\mathrm{t}(\mathrm{s})=$ service time for a regular user using a point-to-point channel.

$\mathrm{t}(\mathrm{r})=$ elapsed time for a re-send of connection request.

$\mathrm{t}(\mathrm{b})=$ access time for a user on broadcast channels.

$\mathrm{t}(\mathrm{l})=$ setup time for channel borrowing.

$\mathrm{t}(\mathrm{h})=$ service time for a roaming user in departing cell.

$\mathrm{t}(\mathrm{o})=$ service time for a roaming user in arriving cell.

\section{GLOBAL CONSTRAINTS}

$$
\mathrm{t}(\mathrm{s})=\mathrm{t}(\mathrm{h})+\mathrm{t}(\mathrm{o}) \text {. }
$$

The above model is a direct extension of the multiple cell model which accommodates channel borrowing. Thus, it covers both channel borrowing and hand-off activities in a cell. As described in the event graph, a user enters the event E7 when she is about to roam into the neighbor cell. From this moment, the base station negotiates with the neighbor base station to complete the handoff process. The duration of services used in the departing cell is denoted as $t(h)$ and the duration of services used in the arriving cell is $t(o)$. Thus, the sum of above two durations is the same as the average service time by a regular user. In the model, we also use an event $\mathrm{D}$ to denote the situation where there is no channel available and the connection has to be dropped. 


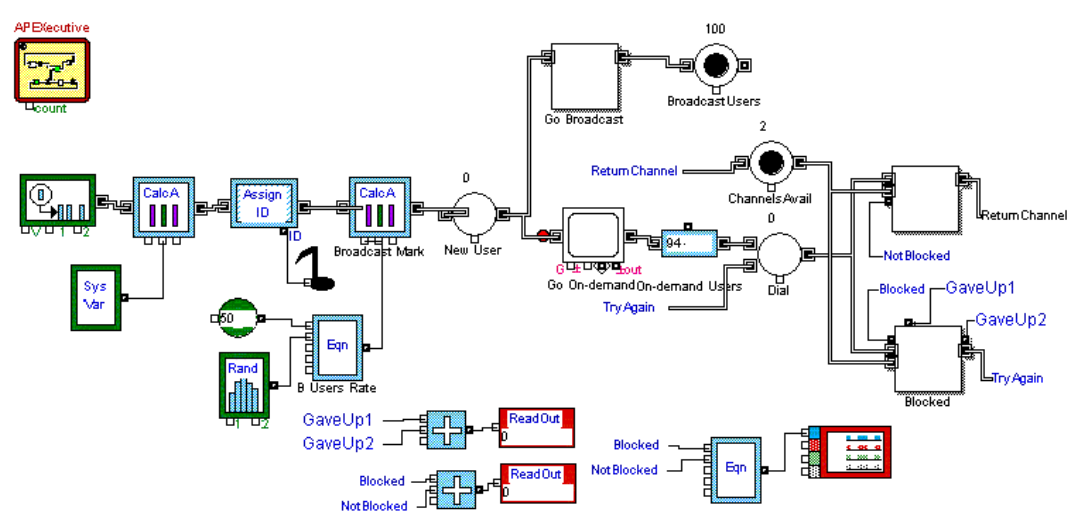

Fig. 5. Extend Model for Single Cell with Broadcast Channel

\section{Simulations and Performance Evaluation}

Blocking probability is one of the major performance measurements for mobile computing systems. If all of the channels are busy in a cell, a service request is blocked. When a large number of mobile users are connected to the system, the blocking probability may reach an unacceptable level. In this situation, the traffic alleviation techniques such as channel borrowing and broadcasting are necessary. In this paper, we use blocking probability to indicate the impact of burst traffic and evaluate the effectiveness of the traffic alleviation techniques.

Our experiments are conducted by using two separate implementation approaches. In order to quickly obtain simulation results, we first use an easy-touse, advanced simulation tool, Extend [Inc95], to develop the simulation systems based on the event graph we proposed earlier. Through Extend's visual interface, we dynamically create simulation models from existing building blocks. Figure 5 shows an Extend simulation mode 2 for one cell system with a broadcast channel. In addition to easy implementation, a great advantage obtained by using Extend is that the activities within the models can be observed through animations during executions. Thus, implementation errors can be easily avoided. However, the performance of our Extend-based simulation systems is rather poor. It took several hours to execute one run of our simulation on a Pentium II/450 PC, so it's not effective to conduct massive simulations for reliability and confidence tests. As a result, we implement another simulation system in Fortran/C on the Pentium PC. Finally, we use the simulation results obtained from these two separate implementations to verify their correctness.

In our experiments, system blocking probability corresponding to different distributions of mobile users arrival rate are obtained. Two distributions of mobile user arrival rate are formulated and shown in Figure 6. In the figure, the

${ }^{2}$ Due to the limited space, we only show the top level presentation of the model.

There are more details in boxes such as 'Go Broadcast'. 


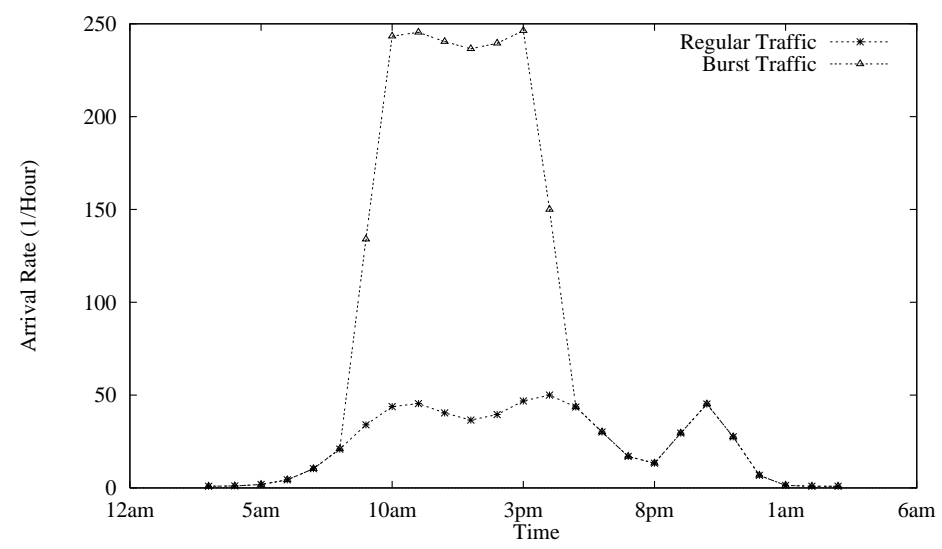

Fig. 6. Distributions of mobile user requests

curves denote the average arrival rate of mobile users during a day. Regular traffic represents the daily distribution of mobile users requesting for services, while burst traffic represents the distribution with some special events occuring, e.g., chess match between Deep Blue and Kasparov. We also compare the blocking probability based on different combinations of channel numbers and service time.

In the current state of our implementation, we have only finished the developments for single cell systems 3 We have configured our systems based on two different channel allocations: one allocates all of the channels to point-to-point communications and the other one allocates one channel for broadcast service and leaves the rest of channels for point-to-point communications.

Monte Carld simulations are conducted on these two implementations described above. In our simulations, we assume that there are 20 channels within a cell. The users have an average service time of 20 minutes. Among blocked users, $15 \%$ of the users try to reconnect. These users have an average delay of 3 minutes for re-tries and they give up after 10 tries. For the system with broadcast channels, we assume that $15 \%$ of users are only interested in the information available on broadcast channels, so those users may tune into the broadcast channel when they enter the system. The systems simulate the activities within a cell during a period of 24 hours. We choose to have the period start at $3 \mathrm{am}$, because a cell usually has the least users at the time.

Figure 7 and 8 show our simulation results on cumulative blocking probability during a day. A point at position $(x, y)$ denotes that there are $y \%$ of mobile users blocked from $3 a \mathrm{am}$ to $\mathrm{x}$. Figure 7 shows the results collected during one run of simulation on Extend system, while Figure 8 shows the average blocking

3 The extensions of simulations for multiple cells environments will be added to the next versions of our implementation.

${ }^{4}$ Monte Carlo methods refers the branch of experimental mathematics that deals with experiments on random numbers. 


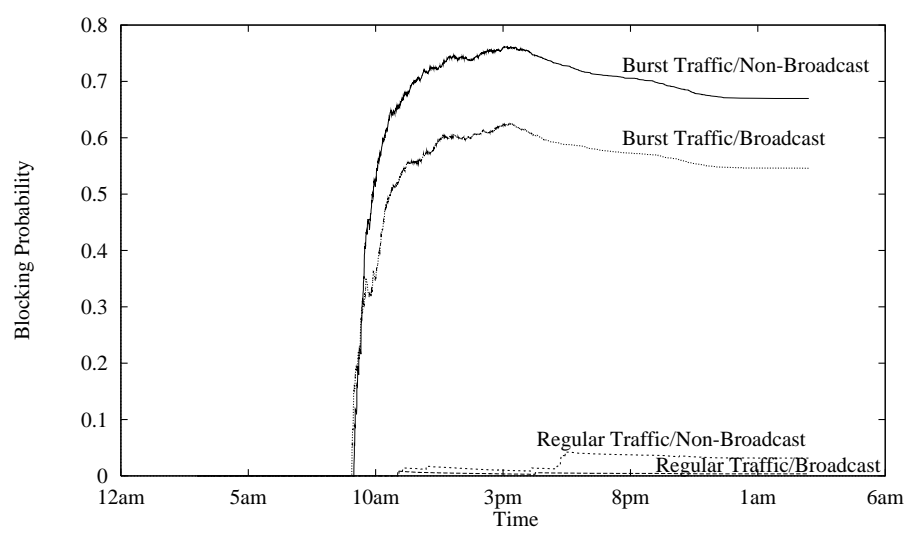

Fig. 7. Blocking probability for a day (Extend).

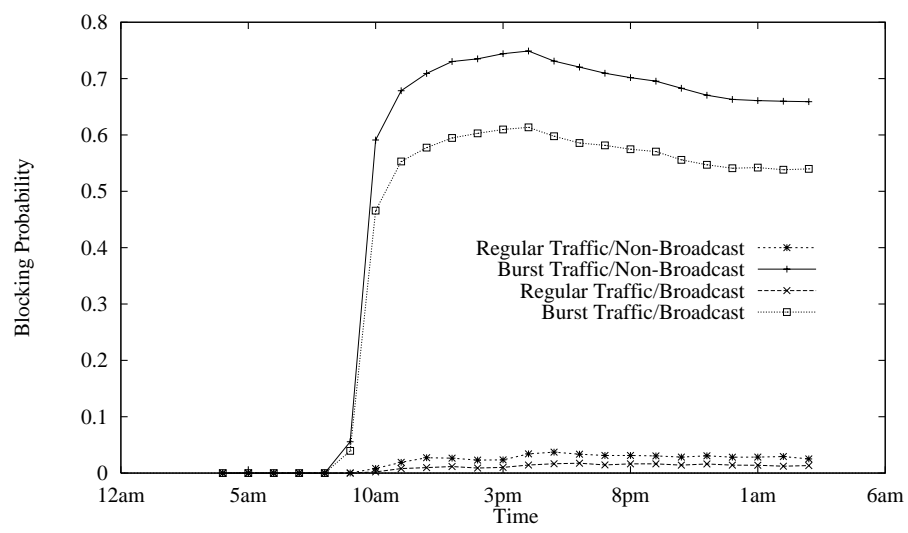

Fig. 8. Blocking probability for a day (C/Fortran).

probability obtained from executing 100 runs of the monte carlo simulations on the $\mathrm{C} /$ Fortran system. It can be easily observed from the figures that simulations on both systems show consistent results, which makes us feel very confident about the correctness and accuracy of our implementations.

From the figures, we can observe that the blocking probabilities for both regular and burst distributions in the first 5 hours are almost 0 since there are virtually no mobile users turned away. For the rest of the day, the blocking probability for regular distribution is kept low while the blocking probability for burst traffic jumps at around the 9am. The blocking probability for burst traffic decreases since the user arrivals are back to normal after 5pm. From the figures, it's also obvious that the blocking probabilities for mobile systems with a broadcast channel are lower than that for systems without a broadcast channel. 


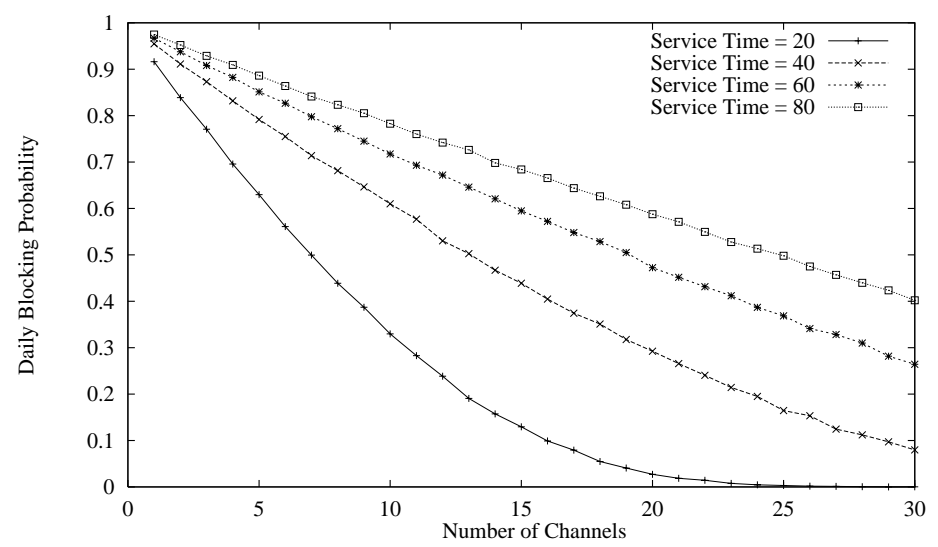

Fig. 9. Blocking probability vs number of channels/service time (nonbroadcasting).

In fact, the blocking probability will be reduced even more significantly if most of the users in the burst traffic are using a broadcast channel. Thus, when a cell is experiencing overwhelming requests for connection due to some timely events, the base station should allocate channels for broadcasting information related to these events in order to serve the burst requests and relieve the load of point-to-point connections.

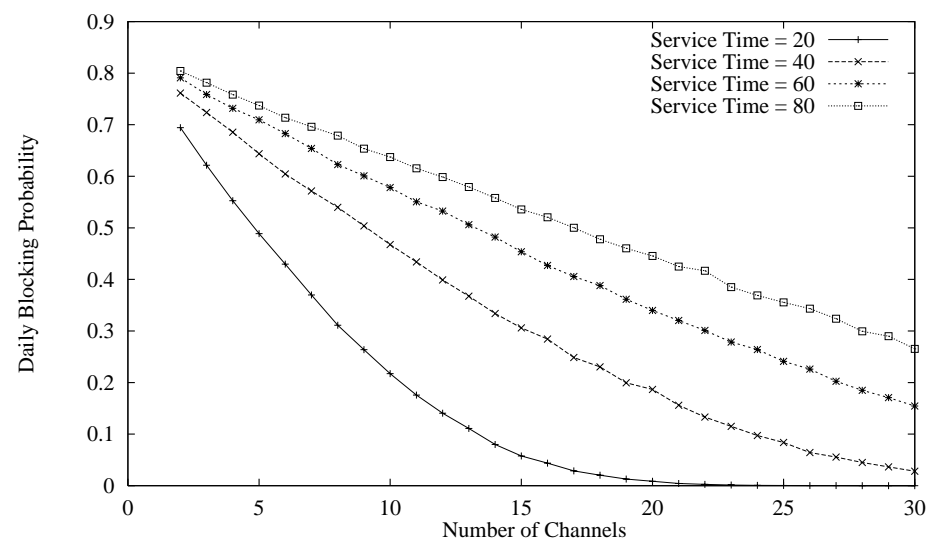

Fig. 10. Blocking probability vs number of channels/service time (broadcasting).

In order to answer questions such as "Given an average service time, how many channels are needed to provide satisfactory services (in terms of blocking probability)?", we conduct simulations to show the daily blocking probability 
corresponding to number of channels and service time (in minutes), for mobile systems without and with a broadcast channel, respectively. For this experiment, we ran 100 monte carlo simulations for each combination of number of channel and service time to obtain the daily blocking rate. As shown in Figure 9 and Figure 10, the blocking probability decreases as the number of channels allocated increases. Meanwhile, the blocking probability increases as the user service time increases. Once again, the figures show that the broadcast channel can reduce the blocking probablity significantly.

The above experiment results are important, because they allow us to verify the correctness of our simulations and set up a foundation for more experiments with much more complex mobile systems in the future. Also, the simulation results provide valuable data and guidance for researchers who are interested in the analytical study of the systems.

\section{Conclusion}

The era of ubiquitous information services is forthcoming. With increasing demands for bandwidth, information broadcasting and cell splitting techniques have been proposed to address the bandwidth and communication traffic problems. New information dissemination systems have to take broadcast channels into their designs and consider issues involved in multiple cell mobile environments, such as channel borrowing and hand-off. Performance analysis models and tools may facilitate a fundamental understanding and fine-tuning of these systems. Thus, they are critical for the business success of the systems.

In this paper, we have proposed three dynamic discrete-event based simulation models for a wireless information dissemination system, which includes both broadcast and on-demand services. Compared to previous analytical studies on similar systems, our models relax constraints assumed by queueing theory based models while providing easily understandable logic for simulations. Moreover, our models treat on-demand and broadcast services coherently and capture one of the special activities in communication systems, retry. Finally, and most importantly, our models address important issues in multiple cell environments, such as channel borrowing and hand-off, and thus enable multiple cell performance analysis.

Based on the dynamic discrete-event model we proposed for wireless information dissemination systems in the single cell scenario, we have implemented two simulation systems: one was quickly prototyped with a visual programming simulation tool, Extend, and the other one was programmed in C/Fortran languages. We conducted Monte Carlo simulations for accumulative blocking probability using the two systems we developed. Our experiments show that the pre-allocated point-to-point channels within a cell can easily handle the regular mobile traffic, but it may have problems handling burst traffic. In this situation, broadcast channels disseminating timely information may satisfy some of the connection demands and thus reduce the blocking rate of the system. Finally, our simulations reveal an important relationship between blocking probability 
and various combinations of number of channels and service time. Through the simulations, we may predict how many channels are needed to satisfy users with certain average service time. This data is critical for mobile system planners and service providers.

This work is only a start of our simulation study on wireless information systems. Through our modeling and implementations, we have set up a foundation for more experiments with much more complex systems in various mobile environments. The simulation results provide valuable information and guidance for researchers who are interested in analytical study of the systems. As the next step, we plan to extend our implementations and experiments to the multiple cell environments. The simulation implementations will serve as our test bed to address issues in designing information services for multiple cell environments, such as global broadcast scheduling, service hand-off, multiple cell cache optimization and mobile proxy management.

\section{References}

AAFZ95. S. Acharya, R. Alonso, M. Franklin, and S. Zdonik. Broadcast disks: Data management for asymmetric communications environments. In Proceedings of the ACM SIGMOD Conference on Management of Data, San Jose, California, 1995.

BB95. A. Bakre and B. R. Badrinath. Handoff and systems support for indirect tcp/ip. In 2nd Usenix Symposium on Mobile and Location-Independent Computing, April 1995.

HP89. S.V. Hoover and R.F. Perry. Simulation: a problem-solving approach. Addison-Wesley, New York, 1989.

IB94. T. Imielinski and B. R. Badrinath. Wireless mobile computing : Challenges in data management. Communication of ACM, 37(10), 1994.

Inc95. Imagine That Inc. Extend - Performance Modeling for Decision Support. Imagine That, Inc., CA, 1995.

IV94. T. Imielinski and S. Viswanathan. Adaptive wireless information systems. In proceedings of SIGDBS (Special Interest Group in DataBase Systems) Conference, Tokyo - Japan, October 1994.

LHL99. W.-C. Lee, Q.L. Hu, and D. L. Lee. A study of channel allocation methods for data dissemination in mobile computing environments. ACM/Baltzer Mobile Networks and Aplications (MONET): Special Issue on Resource Management in Wireless Networks, 4(2):117-129, 1999.

SRB97. K. Stathatos, N. Roussopoulos, and J. S. Baras. Adaptive data broadcast in hybrid networks. In Proceedings of the 23rd VLDB Conference, pages 326-335, Athens, Greece, 1997.

TJ91. S. Tekinay and B. Jabbari. Handover and channel assignment in mobile cellular networks. IEEE Communications Magazine, pages 42-46, November 1991. 